\title{
Structural and electronic properties of the martensitic alloys TiNi, TiPd, and TiPt
}

\author{
Y. Y. Ye \\ Center of Analysis and Testing, Wuhan University, Wuhan, China \\ C. T. Chan \\ Physics Department, Hong Kong University of Science and Technology, Clear Water Bay, Hong Kong \\ K. M. Ho \\ Ames Laboratory-USDOE and Department of Physics and Astronomy, Iowa State University, Ames, Iowa 50011
}

(Received 27 January 1997)

\begin{abstract}
The electronic and structural properties for TiNi, TiPd, and TiPt are studied using first-principles total energy calculations. For each alloy, the structural parameters for a few structural phases are found from energy and force calculations. In each case, we found that the ground state martensitic phase $\left(B 19 / B 19^{\prime}\right)$ is indeed lower in energy than the high temperature $B 2$ phase. In all three alloys, the lowering in energy of the $B 19 / B 19^{\prime}$ structure is associated with the electronic properties such as lowering of the density of states at the Fermi level at the Ti site, and the broadening of the transition-metal $d$ bands in the $B 19 / B 19^{\prime}$ phase compared with the $B 2$ phase. [S0163-1829(97)00331-7]
\end{abstract}

\section{INTRODUCTION}

Martensitic alloys represent an important class of advanced materials which exhibit unusual elastic and mechanical behaviors such as pseudoelastic and shape-memory effects. Some of these alloys are already used in "high-tech", products such as robotic arms, separation and cracking devices, dampers, fastening devices; as well as daily consumer appliances such as eyeglass frames, orthodontic-arch wires and temperature sensitive actuators in air-conditioners. The underlying mechanism of many exotic behaviors of this class of alloys is a martensitic transformation, which is a type of diffusionless structural phase transformation involving a change in the shape of the unit cell together with atomicscale displacements of positions of the atoms in the lattice. Among the martensitic alloys, the Ti family of binary intermetallics has attracted special attention, and within this family, NiTi is by far the best characterized experimentally. NiTi is used extensively in industry because of its resistance to corrosion and the fact that the martensitic transformation temperature $\left(M_{s}\right)$ is near room temperature. There is experimental evidence that links the electronic structure to the stability for a broad class of Ti-based shape-memory alloys. ${ }^{1}$

In the present calculations, we use first-principles total energy calculations to study the electronic, structural, and cohesive properties of TiNi and related alloys TiPd and TiPt in a number of structural phases. Previous calculations on the band structure and the electronic properties of these alloys were based on atomic positions taken from experimental papers. Only the structural properties of NiTi have been measured carefully by experiments, but even for that system, there are some variations in the reported structural parameters. $^{2}$ To the best of our knowledge, this is the first attempt to determine the structural parameters for these structural phases by force calculations from first principles, and our results agree well with the most recent and accurate experimental data. We will show that if the atomic positions are those that optimize the total energy, there are some interesting correlations between the relative stability of the $B 2 / B 19 / B 19^{\prime}$ phases with the electronic structure.

\section{METHOD OF CALCULATION}

The calculations are done within the local density formalism, ${ }^{3}$ and the Hedin-Lundqvist ${ }^{4}$ form for the local exchange-correlation functional is used. Norm conserving scalar-relativistic pseudopotentials ${ }^{5}$ are used for the constituent elements. The electronic Bloch states are expanded in a mixed basis set, ${ }^{6}$ which consists of both numerical orbitals centered on the atomic sites, augmented by plane waves with up to a kinetic energy cutoff of $15 \mathrm{Ry}$. The numerical orbitals are centered on the atomic sites with the radial dependence given by

$$
f(r)=C d(r)\left\{1-\exp \left[-\alpha\left(r-r_{c}\right)^{2}\right]\right\} \quad \text { for } r<r_{c},
$$

where $d(r)$ is the radial part of the $d$-wave function as found from an atomic calculation using pseudopotentials, and $C$ is a normalization constant. We set $f(r)=0$ for $r>r_{c}$, and the values of $\alpha$ and $r_{c}$ are chosen to optimize the convergence of the total energy at a fixed plane wave cutoff near the equilibrium volume. The charge density, which determines the electronic screening potential and the total energy, are computed from the wave functions using a dual real-space and Fourier-space approach. ${ }^{7}$ For the $B 11, B 19$, and $B 19^{\prime}$ structures, with four atoms per unit cells, optimization of the structure is a time-consuming process involving relaxation over many degrees of freedom: the lattice parameters, tilt angle (for the $B 19^{\prime}$ structure), as well as the atomic positions inside the unit cell. The atomic coordinate relaxations are facilitated by computing the Hellmann-Feynman forces. ${ }^{8} \mathrm{~A}$ Broyden algorithm for estimating and updating the force matrix is used to predict the new atomic coordinates during the relaxation process. The convergence with respect to potential and forces are accelerated by a recently proposed scheme. ${ }^{9}$ 
(a)

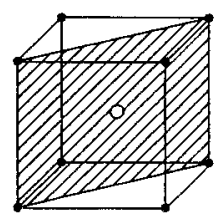

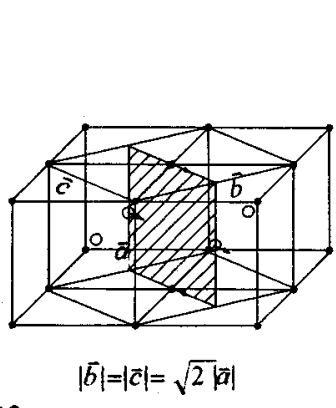

$|\vec{b}|=|\bar{c}|=\sqrt{2}|\vec{a}|$

B2

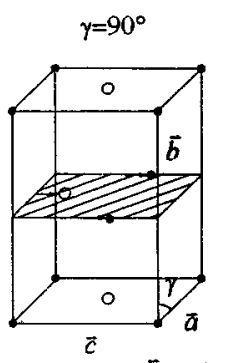

B19 $|\vec{b}|>|\vec{c}| \neq \sqrt{2}|\vec{a}|$

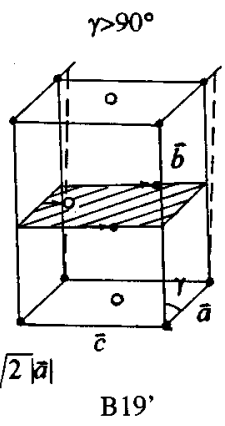

(b)

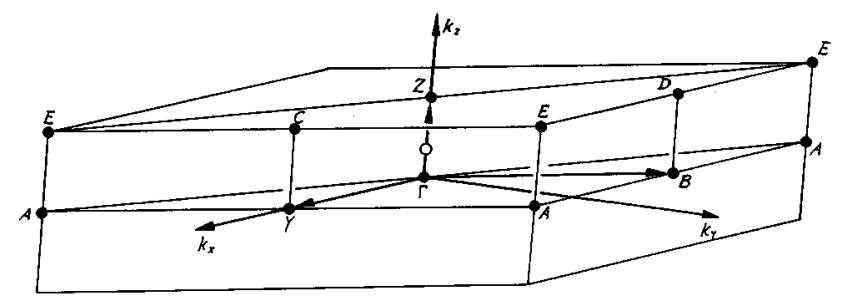

FIG. 1. (a) Unit cells for the $B 2, B 19$, and $B 19^{\prime}$ structures; (b) Brillouin zone for the $B 19^{\prime}$ structure.

\section{NiTi}

The high-temperature phase of $\mathrm{NiTi}$ is a cubic $\mathrm{B} 2(\mathrm{CsCl})$ structure and the low-temperature martensitic phase is a monoclinic $B 19^{\prime}$ structure (see Fig. 1). ${ }^{2}$ The structure of the martensitic phase used to be a subject of some controversy. ${ }^{2,10}$ Although the atomic coordinates of Michal and Sinclar ${ }^{11}$ are quoted most frequently in earlier calculations, and used in previous theoretical calculations on NiTi, we will show below that the atomic parameters of Kudoh et al. $^{12}$ are in better agreement with the optimized atomic positions obtained from total energy calculations and are probably the most accurate structural parameters of $B 19^{\prime}$ NiTi published to date.

In addition to calculating the high-temperature $B 2$ and low-temperature $B 19^{\prime}$ phases of NiTi, we have also calculated the electronic and structural properties of several competing phases that are commonly observed in Ti-based binary alloys: the $B 19, B 11$, and $L 1_{0}$ structures. Equiatomic NiTi has the cubic $B 2$ structure as the high-temperature phase and transforms under cooling to the monoclinic $B 19^{\prime}$ structure via some intermediate rhombohedral structures. ${ }^{2}$ The orthorhombic $B 19$ structure is not observed for NiTi, but is found to be stable for many other Ti alloys such as TiPd, TiPt. ${ }^{2}$ The $B 11$ structure, although not observed for the transition-metal column $\mathrm{Ni}, \mathrm{Pd}$, and $\mathrm{Pt}$, is the stable structure

TABLE I. Structural, elastic, and cohesive properties of NiTi in the $\mathrm{CsCl}$ structure, compared with the experimental data. The elastic constants are in units $10^{12} \mathrm{dyn} / \mathrm{cm}^{2}$.

\begin{tabular}{lcc}
\hline \hline & Present work & Experiment \\
\hline Lattice constant (a.u.) & 5.626 & 5.698 (Ref. 28) \\
Bulk modulus (Mbar) & 1.56 & 1.40 (Ref. 29) \\
Heat of formation (eV) & -0.66 & -0.69 (Ref. 30) \\
$c^{\prime}=\frac{1}{2}\left(c_{11}-c_{12}\right)$ & 0.12 & 0.17 (Ref. 29) \\
$c_{11}$ & 1.68 & 1.62 (Ref. 29) \\
$c_{12}$ & 1.44 & 1.29 (Ref. 29) \\
$c_{44}$ & 0.50 & 0.35 (Ref. 29) \\
\hline \hline
\end{tabular}

for some of the noble metal $\mathrm{Ti}$ alloys such as $\mathrm{TiCu}$ and TiAg. ${ }^{2}$ We will also see that the $L 1_{0}$ phase can be very competitive in energy for TiPd and TiPt.

The calculated structural and cohesive properties of ordered equiatomic NiTi in the $B 2$ structure are compared with experimental data in Table I. These values are determined by fitting the calculated values of the total energy at various volumes to the universal binding curve. ${ }^{13}$ The heat of formation of the NiTi alloy is obtained by subtracting the bulk total energies of the constituent elements ( $\mathrm{Ni}$ in fcc structure, $\mathrm{Ti}$ in hcp structure) from the total energy of the alloy at equilibrium. The reference bulk energy for fcc $\mathrm{Ni}$ is determined from a non-spin-polarized bulk calculation. Including the spin-polarization energy would lower the energy per bulk Ni atom by approximately $0.006 \mathrm{Ry}$ or about $0.082 \mathrm{eV} .^{14}$

The lattice parameters and atomic positions for the various structural phases are found by force and total energy calculations. The crystal structures of the $B 2, B 19$, and $B 19^{\prime}$ phases are shown in Fig. 1. Since these phases are

TABLE II. Structural parameters for the NiTi $B 19$ and $B 19^{\prime}$ phase as determined by the calculation, compared with experimental data for the $B 19^{\prime}$ structure. There are four atoms per unit cell. Only the coordinates of two of the atoms are listed since the coordinates of other pairs are fixed by the inversion symmetry.

\begin{tabular}{lcccc}
\hline \hline & $\begin{array}{c}B 19 \\
\text { Theory }\end{array}$ & $\begin{array}{c}B 19^{\prime} \\
\text { Theory }\end{array}$ & $\begin{array}{c}B 19^{\prime} \\
\text { KTMO (Ref. 12) }\end{array}$ & $\begin{array}{c}B 19^{\prime} \\
\text { MS (Ref. 11) }\end{array}$ \\
\hline$a(\AA)$ & 2.859 & 2.892 & 2.898 & 2.885 \\
$b(\AA)$ & 4.582 & 4.598 & 4.646 & 4.622 \\
$c(\AA)$ & 4.078 & 4.049 & 4.108 & 4.120 \\
$\gamma\left({ }^{\circ}\right)$ & 90 & 97.8 & 97.78 & 96.8 \\
$\operatorname{Ti}(1) x$ & 0.5 & 0.418 & 0.4176 & 0.4726 \\
$\operatorname{Ti}(1) y$ & 0.224 & 0.218 & 0.2164 & 0.2210 \\
$\operatorname{Ti}(1) z$ & 0.25 & 0.250 & 0.25 & 0.25 \\
$\mathrm{Ni}(1) x$ & 0.0 & 0.045 & 0.0372 & 0.0525 \\
$\mathrm{Ni}(1) y$ & 0.683 & 0.674 & 0.6752 & 0.6930 \\
$\mathrm{Ni}(1) z$ & 0.25 & 0.25 & 0.25 & 0.25 \\
\hline \hline
\end{tabular}



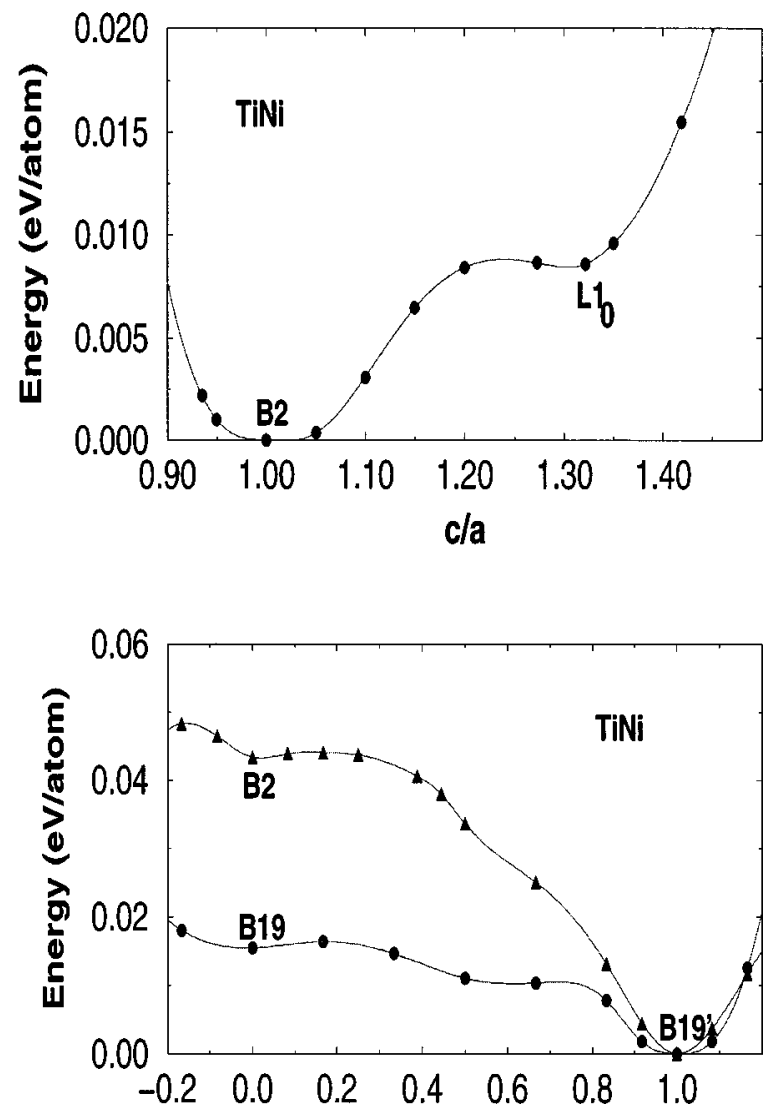

FIG. 2. Energy change as we deform NiTi (A) from the $B 2$ structure to a $L 1_{0}$ structure by varying the $c / a$ ratio; (B) continuously from the $B 2$ structure to the $B 19^{\prime}$ structure and from the $B 19$ to the $B 19^{\prime}$ structure, so that all the structural parameters (lattice parameters and atomic positions) are interpolated linearly from one structure to another. The lines serve as guides to the eye only.

related by martensitic phase transformations, their structures are closely related to one another. If we view the $B 2$ structure as a stacking of (110) planes, then the $B 19$ structure can be formed by displacing the atoms in alternate (110) planes, together with an orthorhombic distortion of the unit cell. The $B 19^{\prime}$ structure is formed by an additional monoclinic distortion. The structural parameters for NiTi in the $B 11, L 1_{0}$, $B 19$, and $B 19^{\prime}$ structures are determined by calculation and in Table II, we compare experimental results for the $B 19^{\prime}$ structure with our calculated $B 19^{\prime}$ and $B 19$ structure. Our results for the $B 19^{\prime}$ structure are closest to the experimental results of Kudoh et al. ${ }^{12}$ Calculation of a $B 19^{\prime}$ structure using the coordinates of MS (Ref. 11) yielded a higher energy structure with significant forces on the atoms in the unit cell. These results indicate that the MS coordinates does not give a good description of the atomic positions in NiTi. ${ }^{15}$ The $B 19^{\prime}$ structure is found to be the most stable among all the structures considered and the relative energies of the structures go in the order $B 11>L 1_{0}>B 2>B 19>B 19^{\prime}$. If we use the $B 19^{\prime}$ structure as a reference, the $B 11, L 1_{0}, B 2$, and $B 19$ structures are $0.17,0.10,0.092$, and $0.024 \mathrm{eV}$ per NiTiformula unit higher than that of the $B 19^{\prime}$ phase. These results differ from the linear muffin-tin orbital (LMTO) calculations of Pasturel et al., ${ }^{16}$ where the $B 19$ structure is higher in energy than the $B 2$ structure and the $L 1_{0}$ structure. One possible reason for this discrepancy is that the atomic coor-

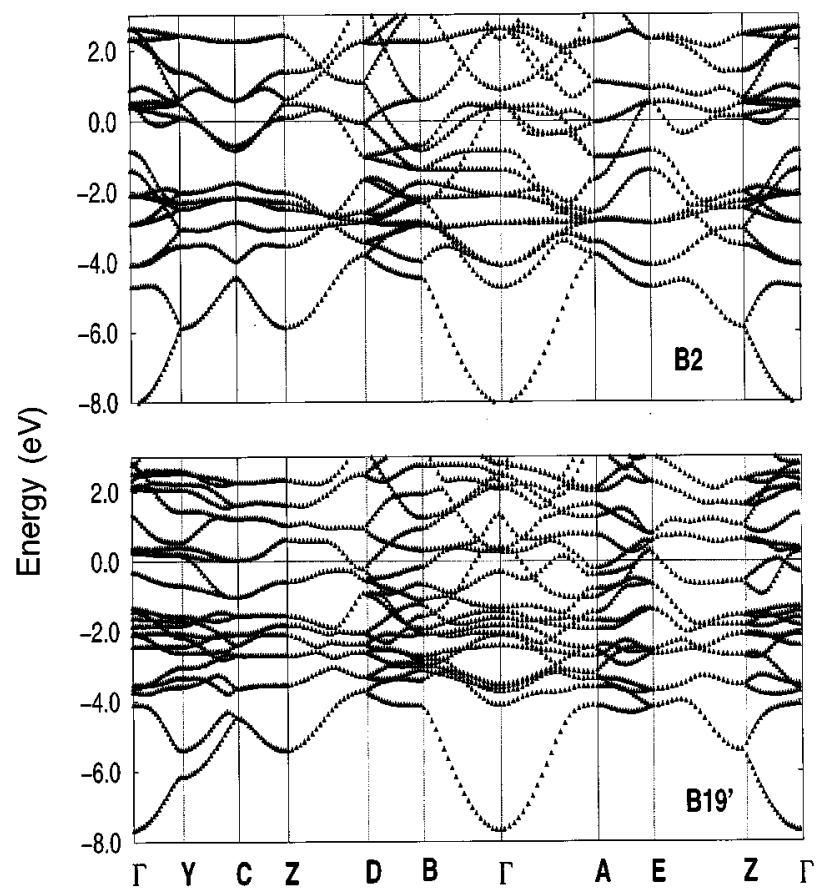

FIG. 3. Band structure of NiTi in $B 2$ and $B 19^{\prime}$ structure. The $B 2$ band structure is folded to the $B 19$ structure Brillouin zone (see text). The Fermi level is at $0 \mathrm{eV}$.

dinates were not optimized in the LMTO calculations: the $B 19^{\prime}$ coordinates were taken from experiments and are close to the minimum as determined by theory. The $B 2$ and $L 1_{0}$ coordinates are fixed by symmetry. However, the coordinates for the $B 19$ structure were taken from AuCd alloys and were not optimal for the NiTi alloy. Therefore the B19 energy becomes higher than the other structure in the LMTO calculations. It is important to use fully relaxed atomic coordinates for reliable energy differences between different structures.

Since martensitic transformations occurs through a cooperative motion of the atomic displacements in the lattice, it is instructive to investigate the energetics of homogeneous deformation from the $B 2$ structure to the $B 19^{\prime}$ structure. To that end, we examine the stability of the $B 2$ structure under unit cell deformation and displacement of atomic positions that can bring a $B 2$ structure to the $B 19^{\prime}$ structure.

We first consider unit cell deformations. The stability against small deformations can be studied by considering the elastic constants. The calculated elastic constants of NiTi in the $B 2$ structure are listed in Table I, and computational details are given in the Appendix. Our results agree well with recent full-potential-linear-augmented plane-wave calculations. ${ }^{17}$ Since $c_{11}, c_{12}, c_{44}$ are all positive, the cubic NiTi $B 2$ structure is stable against orthorhombic distortion of the unit cell towards $B 19$ type unit cell. The elastic constant $c_{11}-c_{12}$, although small, is still positive, which indicates that the $B 2$ structure is stable against shearing along the [110] direction, which means that the $B 2$ structure, viewed as a special $B 19$ structure with higher symmetry, is stable against a monoclinic distortion to the $B 19^{\prime}$ structure. The cubic $B 2$ structure is thus stable against unit cell distortions at $T=0$. To further illustrate the stability of the $B 2$ structure against unit cell distortion, we deform the $B 2$ structure by 


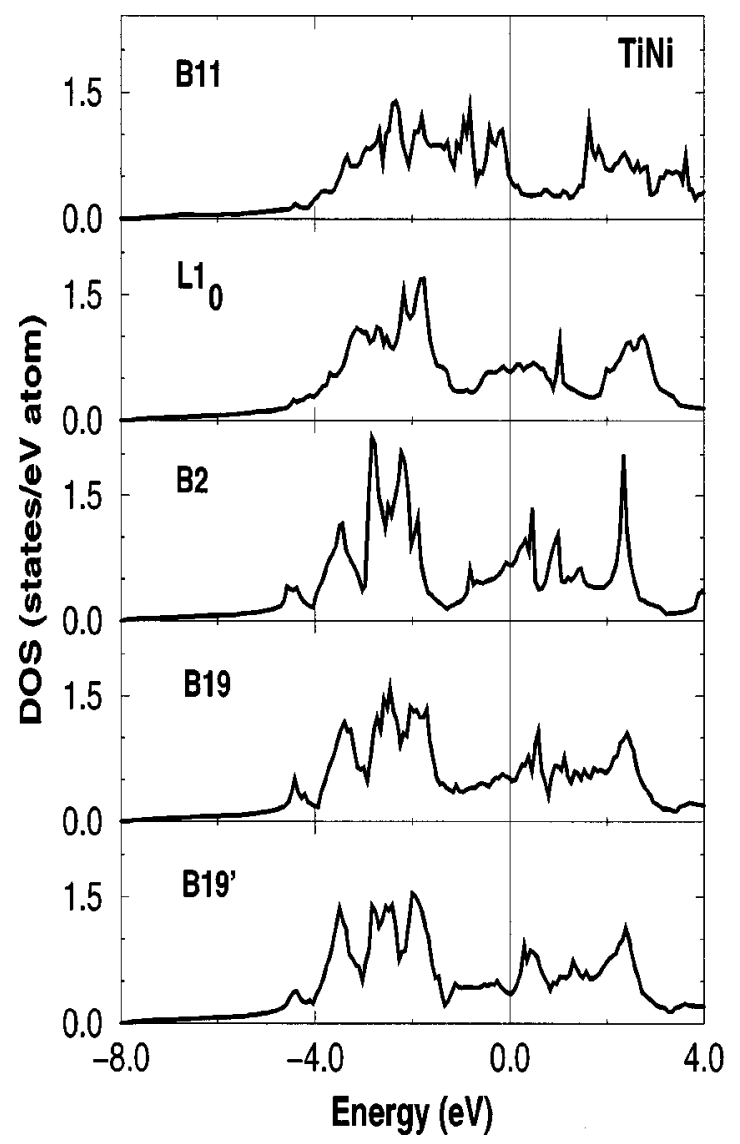

FIG. 4. Density of states of NiTi in various structures, with atomic positions and structural parameters that optimize the total energies. The Fermi level is at $0 \mathrm{eV}$.

continuously varying the $c / a$ ratio, but keeping the volume constant. In this manner, we can deform the $B 2$ structure continuously into a $L 1_{0}$ structure. In the upper panel of Fig. 2 , we show the energy change as we deform the $B 2$ structure to a $L 1_{0}$ structure by varying the $c / a$ ratio from 1 to about $\sqrt{2} .^{18}$ If we regard the $B 2$ structure as a bcc structure with two elements, then the $L 1_{0}$ structure may be regarded as a fct structure with two constituent elements. The optimal $c / a$ ratio for the NiTi $L 1_{0}$ structure is 1.32 and is very close to that of $\mathrm{AuCu}$. It is clear from the figure that the $L 1_{0}$ structure is unfavorable in energy relative to the $B 2$ structure and that the $B 2$ structure is stable against the deformation. Next, we consider atomic displacements ("shuffling" of the atoms) in the NiTi structure. We tried moving the $\mathrm{Ni}$ and $\mathrm{Ti}$ atoms by small incremental displacements that are proportional to the final displacements of the atoms in the NiTi $B 19^{\prime}$ structure, which consists of atomic displacements in alternate (110) layers in the $B 2$ structure, and we found that that the displacement causes an increase in energy if the unit cell is kept cubic. This is consistent with the tight-binding calculation of Zhao et al. ${ }^{19}$ that the zone boundary phonons along the $B 2$ [110] directions are not soft. These results indicate that there is an energy barrier between the cubic $B 2$ structure and the $B 19^{\prime}$ structure under both pure homogeneous lattice deformation or pure atomic shuffle displacements without unit cell deformation. However, we can also investigate what happens when the different degrees of freedom are allowed to couple in a cooperative deformation pro-
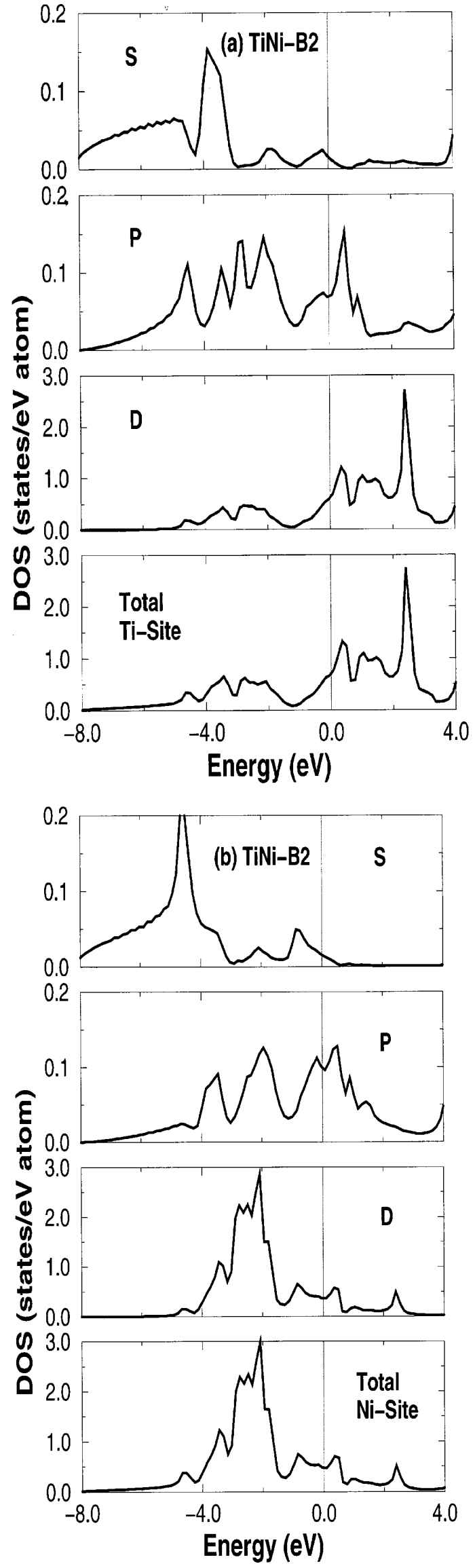

FIG. 5. Site and angular momentum decomposed density of states for TiNi in the $B 2$ structure for (a) Ti site; and (b) Ni site. The scales are not the same in different panels. 
cess: rather than searching for the path with the lowest energy barrier, we examined one in which the structure is deformed continuously from the $B 19$ to the $B 19^{\prime}$ structure, so that all the structural parameters (lattice parameters, atomic positions) are interpolated linearly from one structure to another. The lower panel of Fig. 2 shows the energy change of the crystal as it changes from the $B 2$ to the $B 19^{\prime}$ structure: within the noise of the calculation, there is hardly any barrier. We also show for completeness the energy change as we continuously deform the $B 19$ to the $B 19^{\prime}$ structure. It is quite likely that there are paths in the configuration space that connect the $B 2$ and the $B 19^{\prime}$ structure that have no barrier. This is a manifestation of the cooperative effects of various degrees of freedom, while each can increase the energy, they can combine to lower the energy of the system. In addition to its instability towards the $B 19^{\prime}$ phase, we note that the $B 2$ structure is also ustable towards distortions corresponding to a transverse phonon along the $B 2$ [110] direction close to the wave vector $(1 / 3$, $1 / 3,0)(2 \pi / a) .{ }^{19}$ This instability leads to the formation of the $R$ phase which is an intermediate phase formed for some stoichiometric compositions when the $B 2$ structure is cooled. $^{2}$

\section{ELECTRONIC STRUCTURE}

In this section, we examine the electronic structure associated with the $B 2$ and $B 19^{\prime}$ structure of $\mathrm{NiTi}$, and will correlate the relative structural stability with the electronic structure. The band structures of both the $B 2$ and $B 19^{\prime}$ structures has been considered previously, ${ }^{20,21}$ where the atomic positions are based on earlier published coordinates. ${ }^{15}$

In Fig. 3, we plot the band structure of NiTi in the $B 2$ and $B 19^{\prime}$ structures along high symmetry lines. The band structures correspond to the structural parameters that optimize the total energy within our first-principles total energy scheme. Since the $B 19^{\prime}$ structure has four atoms per unit cell, while $B 2$ has two atoms per cell, the Brillouin zone (BZ) of $B 19^{\prime}$ is about twice as big. While the $B 2$ structure has a simple cubic BZ, the monoclinic $B 19^{\prime}$ structure has a more complicated Brillouin zone, shown in Fig. 1. To compare the band structure of the two phases, we fold the $B 2$ band structure to a $B 19$ Brillouin zone; i.e., instead of choosing (in units of $2 \pi / a$ ) a primitive cubic unit cell with reciprocal lattice vectors given by $\vec{b}_{1}(0,0,1), \quad \vec{b}_{2}=(0,1,0)$, $\vec{b}_{3}=(0,0,1)$, we choose an orthorhombic unit cell with reciprocal lattice vectors given by $\vec{b}_{1}^{\prime}=(0,0,1), \vec{b}_{2}^{\prime}=(0.5,0.5,0)$, $\vec{b}_{3}^{\prime}=(0.5,-0.5,0)$. In this case, each $k$ point in the $B 19 /$ $B 19^{\prime}$ structure will correspond to two inequivalent $k$ points in the $\mathrm{BZ}$ of the $B 2$ structures. For example, if $\vec{k}=x_{1} \vec{b}_{1}^{\prime}$ $+x_{2} \vec{b}_{2}^{\prime}+x_{3} \vec{b}_{3}^{\prime}$ where $\vec{b}_{1}^{\prime}, \vec{b}_{2}^{\prime}, \vec{b}_{3}^{\prime}$ are for the $B 19$ structure, the corresponding $2 k$ points in the $B 2$ structure are given by $\vec{k}=0.5\left(x_{2}+x_{3}\right) \vec{b}_{1}+0.5\left(x_{2}-x_{3}\right) \vec{b}_{2}+x_{1} \vec{b}_{3}$ and $\vec{k}=0.5\left(x_{2}\right.$ $\left.+x_{3}-1\right) \vec{b}_{1}+0.5\left(x_{2}-x_{3}-1\right) \vec{b}_{2}+x_{1} \vec{b}_{3}$. When we compare the two band structures, we see that there is generally less degeneracy in the $B 19^{\prime}$ band structure, due to the fact that the $B 19^{\prime}$ structure has a lower symmetry. Although the band dispersions are rather similar at lower energies, there are significant differences near the Fermi level $\left(E_{F}\right)$ which will
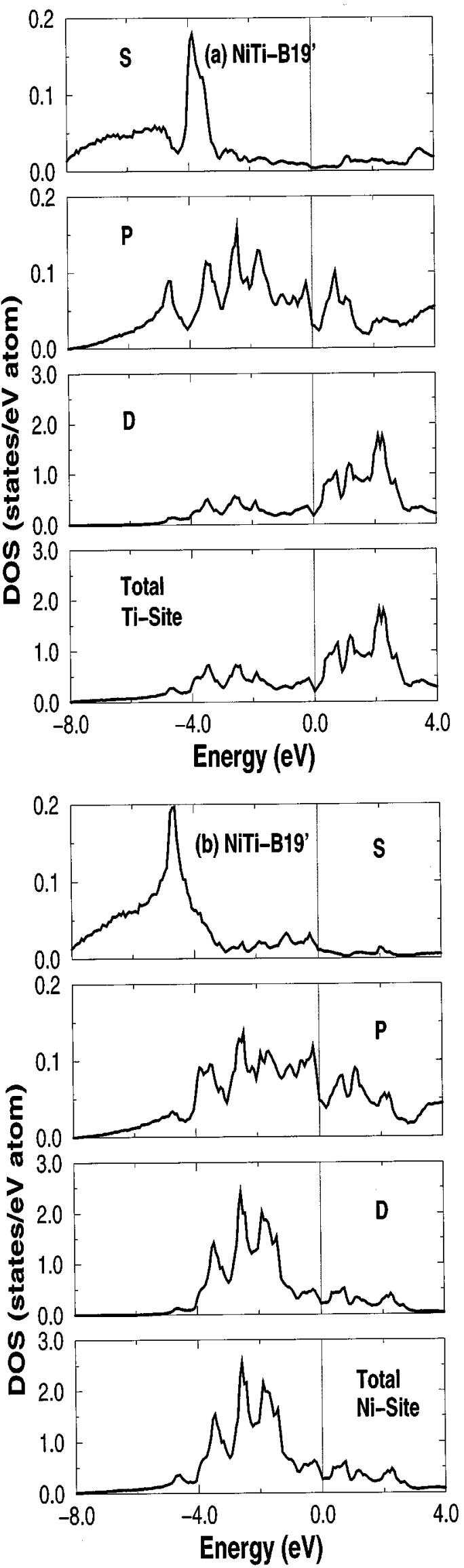

FIG. 6. Site and angular momentum decomposed density of states for TiNi in the $B 19^{\prime}$ structure for (a) Ti site; and (b) Ni site. The scales are not the same in different panels. 
TABLE III. Calculated structural parameters of TiPd and TiPt in the $B 19$ structure, compared with experimental results. The $\mathrm{Pd} / \mathrm{Pt}$ positions are given by $\left(0, y_{X}, 0.25\right)$ where $X$ is either $\mathrm{Pd}$ or $\mathrm{Pt}$, and the $\mathrm{Ti}$ positions are given $\left(0.5, y_{\mathrm{Ti}}, 0.25\right)$, and their inversion positions.

\begin{tabular}{lcccc}
\hline \hline & TiPd & TiPd & TiPt & TiPt \\
\hline & Theory & Expt. & Theory & Expt. \\
\hline$a(\AA)$ & 2.79 & 2.81 (Ref. 31), 2.78 (Ref. 25) & 2.81 & 2.76 (Ref. 31), 2.73 (Ref. 25) \\
$b(\AA)$ & 4.81 & 4.89 (Ref. 31), 4.86 (Ref. 25) & 4.83 & 4.84 (Ref. 31), 4.79 (Ref. 25) \\
$c(\AA)$ & 4.52 & 4.56 (Ref. 31), 4.55 (Ref. 25) & 4.55 & 4.59 (Ref. 31), 4.55 (Ref. 25) \\
$y_{X}$ & 0.689 & & 0.688 & \\
$y_{\mathrm{Ti}}$ & 0.201 & & 0.197 & \\
\hline \hline
\end{tabular}

affect various physical properties such as susceptibilities and optical conductivities in the two phases.

To better examine the differences in electronic structures of the different crystal structures, we plot the various density of states (DOS) in Fig. 4, calculated with the lineartetrahedron method. ${ }^{22}$ In addition to the $B 2$ and $B 19^{\prime}$ structure, we also show for comparison the DOS of the other structures we have calculated. The DOS is expressed in number of states per atom per energy interval. The DOS of the $B 2$ structure resembles that of a bcc metal in that it consists of two sets of peaks separated by a dip about $1.5 \mathrm{eV}$ wide. The lower energy occupied set of peaks come mainly from the Ni $d$ states, and the higher energy peaks are mainly due to the $d$ states of Ti. The Fermi level $E_{F}$ lies in a small dip in a rising part of the DOS below the higher energy peaks which are mostly unoccupied.

Comparing with other structures, we found that while the DOS for the $B 11$ and $L 1_{0}$ structure are quite different from the $B 2$ structure, the DOS for the $B 19$ and $B 19^{\prime}$ structures are rather similar to that of the $B 2$ structure. In general, the peaks in the $B 19$ and $B 19^{\prime}$ structures tend to be broader than the $B 2$ structure, mainly because of the lowering of symmetry. There are some changes in a range of $\pm 1.5 \mathrm{eV}$ around the Fermi level. We notice that the dividing dip of the DOS at about $-1.5 \mathrm{eV}$ in the $B 2$ structure becomes less conspicuous in the $B 19 / B 19^{\prime}$ structures and the rising DOS from -1.5 to $0.5 \mathrm{eV}$ is now replaced by a flatter plateau with a smaller DOS. There is also a significant lowering of the DOS at $E_{F}$. For $B 19 / B 19^{\prime}$, the DOS has a minimum (a dip) at $E_{F}$. This dip is most pronounced when the atoms have relaxed to their most stable positions in the unit cell. If the atomic positions are displaced so that they are not those that optimize the total energy of the system, we find that the $E_{F}$ does not correspond to the dip. We can also see that the dip of the $B 19^{\prime}$ structure is more conspicuous than that of the $B 19$ structure. If we compare our calculated $B 19^{\prime}$ DOS (based on atomic coordinates that optimize the total energy) with previous calculations, we found that ours agrees well with those that were computed with correctly reported experimental coordinates. ${ }^{20,16}$ On the other hand, our results have a noticeable deviation, especially at and near $E_{F}$, from those that had possibly employed incorrect atomic coordinates. $^{21}$

A further investigation into the electronic structure changes accompanying the $B 2-B 19^{\prime}$ structural transition can be studied by decomposing the density of states into site and angular momentum contributions. ${ }^{23}$ The results are shown in Fig. 5 for the $B 2$ structure, and Fig. 6 for the $B 19^{\prime}$ structure. For both $\mathrm{Ni}$ and Ti sites, the DOS is dominated by contributions from the $d$ states. We observe that the DOS below $E_{F}$ are mostly $d$ states contributed by the $\mathrm{Ni}$, while the DOS above $E_{F}$ are mainly $d$ states in the Ti sites. There is, however, a significant intensity for $\mathrm{Ti}$ site at the lower energies and $\mathrm{Ni}$ site at the higher energies indicative of hybridization and some covalent bonding between the orbitals on the two sites. In the $B 2$ structure, the DOS at $E_{F}$ is due to the Ti $d$ states, the $\mathrm{Ni} d$ states, the Ni $p$ states and the Ti $p$ states in order of importance. There are negligible contributions from the $s$ states. In going to the $B 19^{\prime}$ structure, the most dramatic changes in the DOS occur near the Fermi level, within a range of $\pm 1.5 \mathrm{eV}$ : there is an upward shift of the Ti $d$ - and $p$-states just above $E_{F}$ by about $0.5 \mathrm{eV}$ and a downward shift in the Ni $p$ states by about $0.8 \mathrm{eV}$. This results in the opening up of a gap between some of the energy bands near $E_{F}$ leading to a depletion of the total DOS at $E_{F}$. In addition, there is an increase of bandwidth of the $\mathrm{Ni}$ $d$ states, signaling a stronger participation of the $d$ states of $\mathrm{Ni}$ in the bonding in the $B 19^{\prime}$ structure. We believe that both the lowering of the DOS at $E_{F}$ and the stronger bonding of

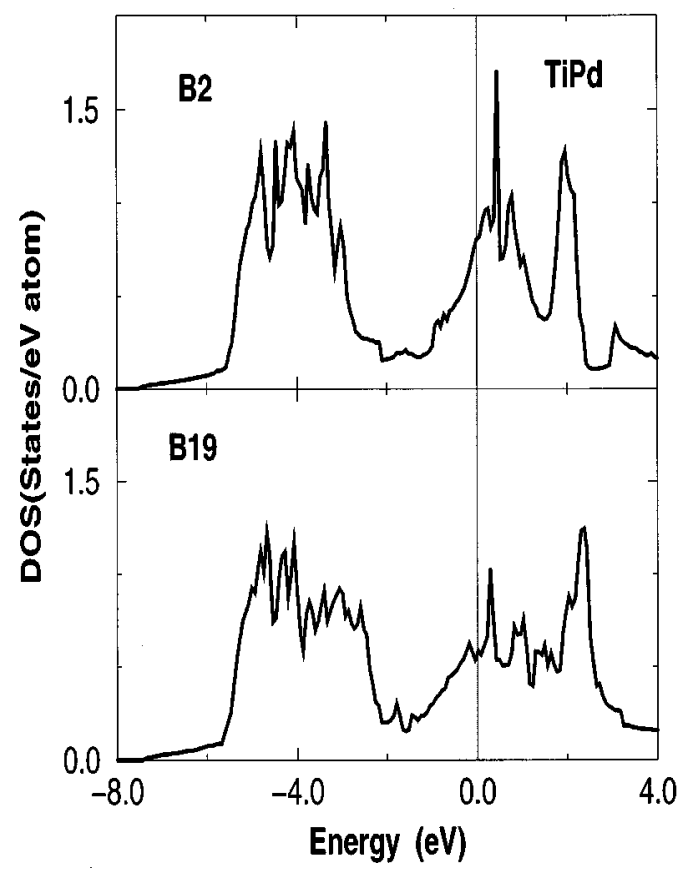

FIG. 7. Total density of states of TiPd in the $B 2$ and $B 19$ structures, with structural parameters that optimize the total energies. 


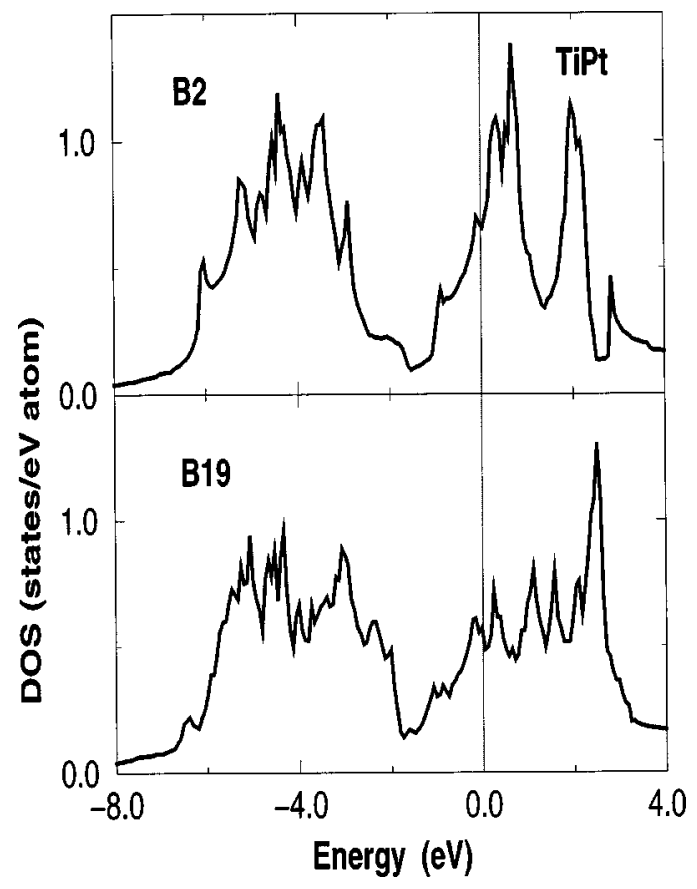

FIG. 8. Total density of states of TiPt in $B 2$; and $B 19$ structures, with structural parameters that optimize the total energies.

the Ni $d$ state are contributing to the stability of the $B 19^{\prime}$ structure relative to the $B 19$ structure. The difference of the angular momentum decomposed local density of states (LDOS) between the $B 19$ and $B 19^{\prime}$ structure is fairly small, mainly a further depletion of the DOS at $E_{F}$ for the $p$ and the $d$ channel at both the $\mathrm{Ti}$ and the Ni site for the $B 19^{\prime}$ structure, which may account for its extra stability.

Ternary alloying additions affect the transformation temperature of $\mathrm{NiTi}^{24}{ }^{24}$ and there is no simple theory that can explain all the observations. Within a rigid-band picture, and assuming that the DOS at $E_{F}$ is an important indication of the stability of the alloy, we expect that a small amount of impurities will destabilize the $B 19$ structure since the $E_{F}$ is at a dip and whether we increase or decrease the electrons will increase the energy. However, the DOS at $E_{F}$ for the $B 2$ structure is quite high and is located on a rising slope of a peak in the DOS, so that impurities that reduce the total number of electrons may stabilize the $B 2$ while those that increase the number of electrons can destabilize it further compared with the $B 19^{\prime}$ structure. This simple picture is consistent with the experimental observation that $M_{s}$ is decreased if $\mathrm{Ni}$ is replaced by $\mathrm{Co}$ or $\mathrm{Fe}$, which have less electrons than $\mathrm{Ni}$, and $M_{s}$ is increased only when $\mathrm{Ni}$ is replaced by $\mathrm{Pd}$ and $\mathrm{Au}$, which have equal or more electrons than $\mathrm{Ni}$. However, the rigid-band picture cannot explain why $M_{s}$ is decreased if $\mathrm{Ti}$ is replaced by elements such as $\mathrm{V}, \mathrm{Cr}, \mathrm{Mn}$. This picture neglects changes in the electronic structure with the substitution of new elements and relaxation of the atomic positions with substitution caused by the martensitic transformation. A more detailed model going beyond the simple rigid-band picture is probably required for a comprehensive description of the effects of alloy substitution.

\section{TiPd AND TiPt}

In the following, we will examine the structural end electronic properties of TiPd and TiPt. The lattice constants of
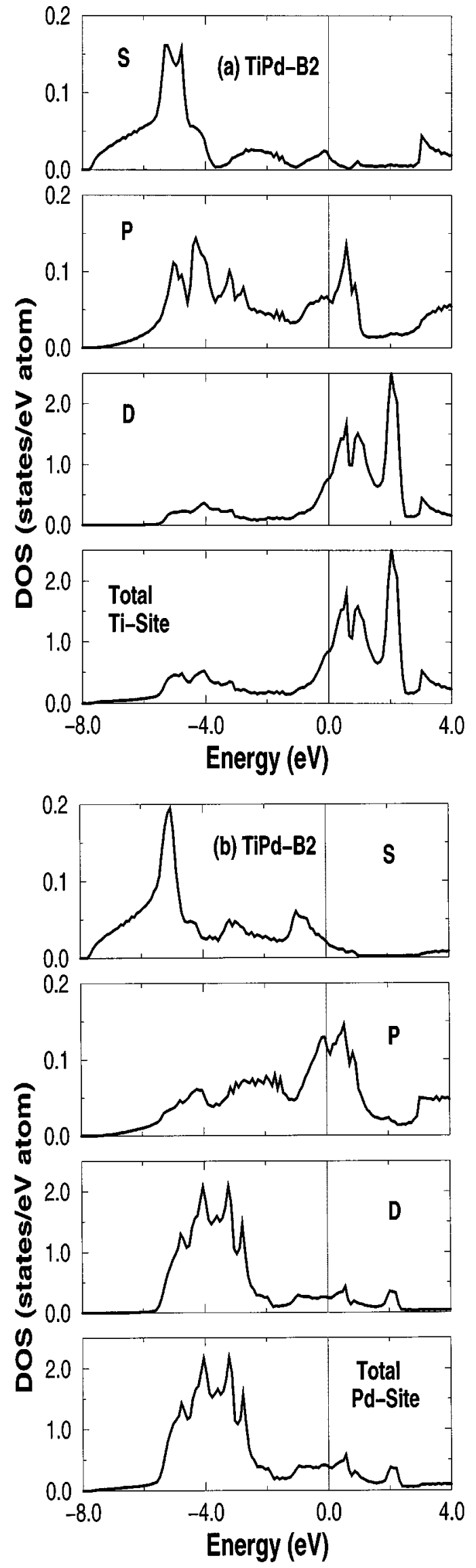

FIG. 9. Site and angular momentum decomposed density of states for TiPd in the B2 structure for (a) Ti site; and (b) Pd site. The scales are not the same in different panels. 

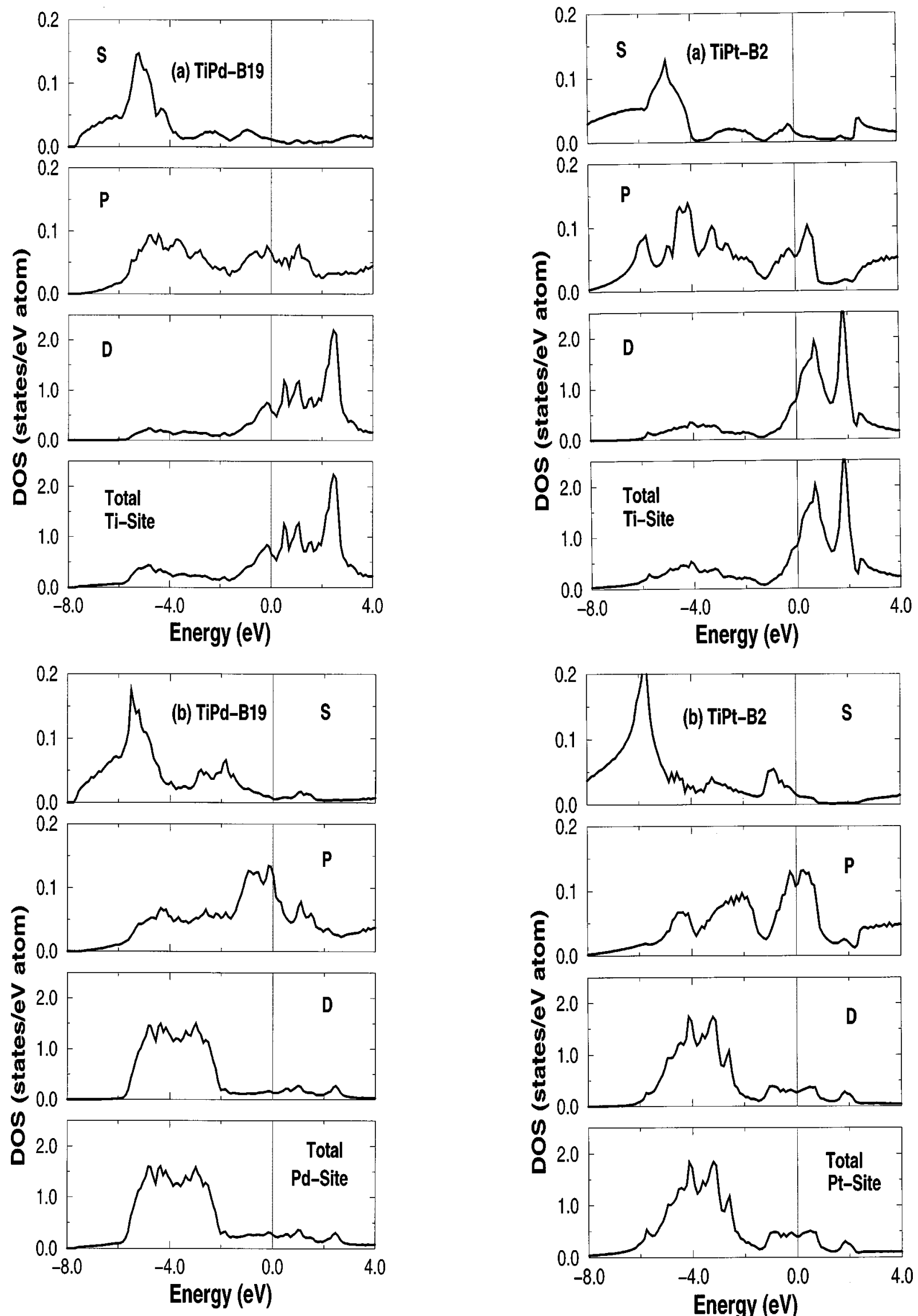

FIG. 10. Site and angular momentum decomposed density of states for TiPd in the B19 structure for (a) Ti site; and (b) Pd site. The scales are not the same in different panels.

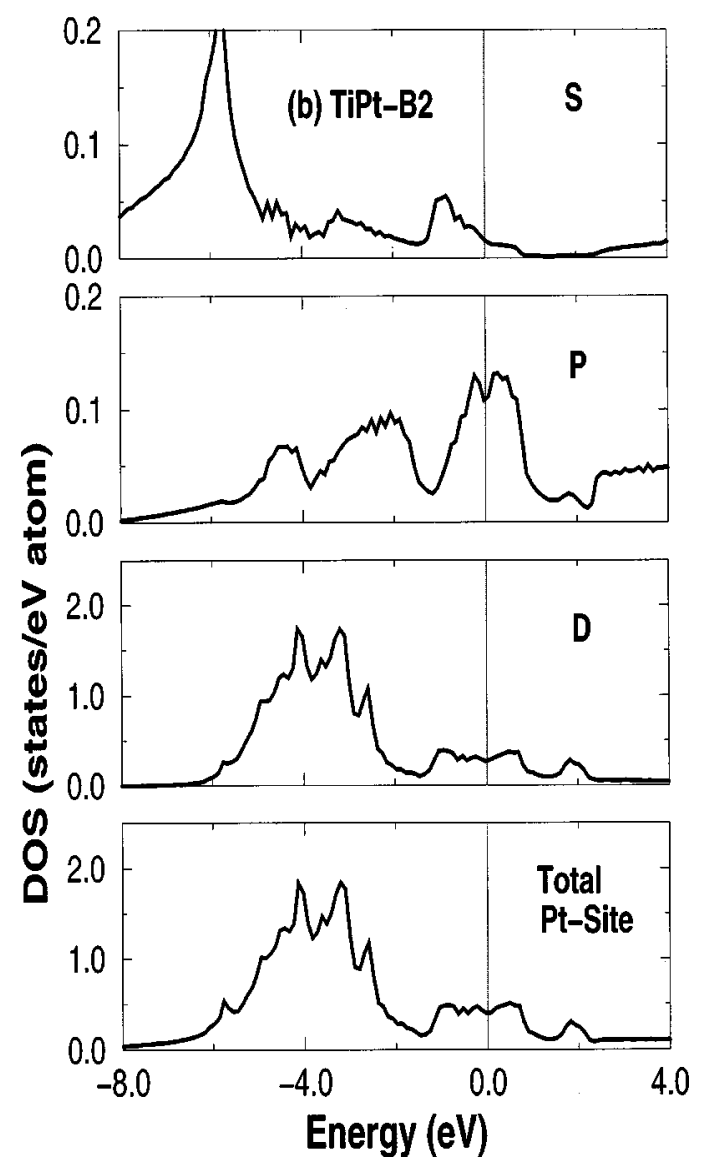

FIG. 11. Site and angular momentum decomposed density of states for TiPt in the B2 structure for (a) Ti site; and (b) Pt site. The scales are not the same in different panels. 
the $B 2$ phase of TiPd and TiPt are found to be $3.14 \AA$ and $3.15 \AA$, respectively, compared with experimental values of $3.18 \AA$ and $3.17 \AA$. We note that the lattice constants are about the same for TiPd and TiPt, and bigger than that of NiTi. This is consistent with the fact that the lattice constants of bulk Pd and Pt are about the same and bigger than that of Ni.

Experimentally, both alloys are found in the $B 19$ structure at low temperatures. One way to view the $B 19$ structure is to view the (110) planes of the $B 2$ structure as the (100) basal plane of the $B 19$ structure. The unit cell has an orthorhombic distortion, and atoms are displaced from their high symmetry positions in the $B 2$ structures. The lattice parameters and the atomic positions as determined by force calculations are listed in Table III for TiPd and TiPt. Although the lattice parameters of TiPd and TiPt in the B19 structure has been reported, ${ }^{25}$ we are not aware of experimental determination of the atomic positions. Previous calculations on the electronic structure of the $B 19$ phase of these alloys seemed to have adopted the coordinates of $\mathrm{CdAu}$, which need not be appropiate for TiPd and TiPt. In the coordinate systems we have chosen, the $\mathrm{Ti}$ atoms should be at $(0.5,0.25,0.25)$ and the $\mathrm{Pd}$ (or Pt) atoms at $(0.5,0.75,0.25)$ and their inversion images from the origin if there are no atomic displacements from the $B 2$ structure. We see from Table III that the atomic displacements correspond to relative shifting of alternate (110) planes in the $B 2$ structure. However, the (110) planes in the $B 2$ structure are not rigid in the shifting process, because there is a displacement of the $\mathrm{Ti}$ atoms relative to the $\mathrm{Pd}$ (and Pt) atoms. The atomic displacements are thus a bit more complex than those given in the description by Otsuka et al. ${ }^{10}$ which only involves relative displacement of rigid (110) planes in the $B 2$ structure.

When we compare the energy differences between different TiPd structural phases, we found that the relative energies go in the order $B 2>B 11>L 1_{0}>B 19$; and if we use the ground state $B 19$ structure as a reference, the $L 1_{0}, B 11$, and $B 2$ structures are $0.03 \mathrm{eV}, 0.09,0.19 \mathrm{eV}$ per formula-unit higher in energy. For the case of TiPt, the relative energies go in the order $B 11>B 2>L 1_{0}>B 19$, and the energies of the $B 11, B 2$, and $L 1_{0}$ structures are $0.36,0.31,0.03 \mathrm{eV}$ per formula-unit higher than the $B 19$ structure. We note that the ordering of energy for various structural phases is not the same as for the case of TiNi. For TiNi, structural phases such as $L 1_{0}$ and $B 11$ that are not found experimentally for TiNi all have higher energies than the $B 2$ structure. However, for both TiPd and TiPt, the $B 2$ structure actually has higher energy than some of these phases that were not observed. This is consistent with the FLAPW results of Bihlmayer et al. ${ }^{26}$ who considered TiNi and TiPd in the $B 2$, $B 19 / B 19^{\prime}$, and $L 1_{0}$ structures. Our TiPt heat of formation in the $B 2$ phase and the difference in heat of formation between the $B 2$ and $B 19$ structure agree well with the full-potentiallinearized-augmented-Slater-type orbital calculations of Fernando, Watson, and Weinert. ${ }^{27}$ Interestingly, $B 19^{\prime}$ metastable phases can be found for both TiPd and TiPd, with a small tilting of the $B 19$ unit cell. The $B 19^{\prime}$ phase of TiPd is about $0.04 \mathrm{eV} /$ formula-unit higher in energy than the orthorhombic $B 19$ structure, but for TiPt, the $B 19^{\prime}$ structure with a tilt angle of about $3.8^{\circ}$ has almost the same energy as the $B 19$ structure. Since the energy is competitive, it would be interesting to reexamine the experimental data to see if the $B 19^{\prime}$ structure actually coexists with the $B 19$ structure for TiPt at low temperatures.

The total density of states for TiPd and TiPt in the $B 2$ and $B 19$ structures are compared in Figs. 7 and 8, respectively. For both alloys, the most obvious change in the total DOS in going from the $B 2$ to the $B 19$ structure is the significant decrease in the DOS at $E_{F}$, and the increase of bandwidth for the $d$ bands below $E_{F}$. Similar to the case of TiNi, the total DOS for the $B 2$ structures in both systems are two groups of states separated by a wide pseudogap centered at about $2 \mathrm{eV}$ below $E_{F}$, with the lower part being mainly contributions from the transition metal $(\mathrm{Pd} / \mathrm{Pt})$ and the upper part mainly states from the $\mathrm{Ti}$ site. It is quite obvious from the lower panel of these figures that in the $B 19$ structure, the dividing pseudogap between the transition-metal states and the Ti states becomes smaller and there is an increase in the bandwidth in states on either side of the dividing gap, signaling a stronger bonding between $\mathrm{Pd} / \mathrm{Pt}$ with the Ti.

The site and angular momentum decomposed DOS for TiPd and TiPt in $B 2$ and $B 19$ structures are shown in Figs. 9-12, respectively. The overall behavior of TiPd and TiPt is quite similar to that of TiNi. The transition from the $B 2$ to the $B 19$ structure is accompanied by a depletion of states near $E_{F}$ caused by a downward shift of the Pd and Pt $p$ states, an upward shift in the unoccupied Ti $d$ states, as well as an increase in bandwidth of the $d$ states of both constituents.

\section{HEAT OF FORMATION AND THE $M_{s}$ TEMPERATURE}

From the last two columns in Table IV, we see that there is an obvious correlation between the $M_{s}$ temperature and the $\Delta H$, defined as the heat of formation of the martensitic phase ( $B 19$ or $B 19^{\prime}$ ) minus that of the $B 2$ phase. This is not surprising: A small difference in the heat of formation between the autensite and the martensite indicates that the $B 2$ structure is not that unstable relative to the low-temperature phase and can thus survive to a lower temperature, leading to a lower $M_{s}$ temperature. We also note that for the three $\mathrm{Ti}$ alloys we have studied, the calculated $\Delta H$ is more or less proportional to the calculated heat of formation of the $B 2$ phase. Therefore, the $M_{s}$ temperature is also correlated with the heat of formation of the $B 2$ phase such that the alloy with a larger $B 2$ phase heat of formation has a higher $M_{s}$. We also see from Table IV a direct correlation between the melting temperature and the heat of formation of the $B 2$ phase. These correlations are of course observed for an isoelectronic series, where the number of valence electrons in the alloys remains the same and the electronic structure are rather similar, so that the difference is mainly a matter of the energy scale. Such observations may not hold if we are moving across the Periodic Table so that the total number of electrons changes.

\section{DISCUSSION AND SUMMARY}

We used first-principles total energy calculations to provide an accurate description of the structural and electronic properties of equiatomic TiNi, TiPd, and TiPt alloys. The structural parameters of these alloys were obtained by the 

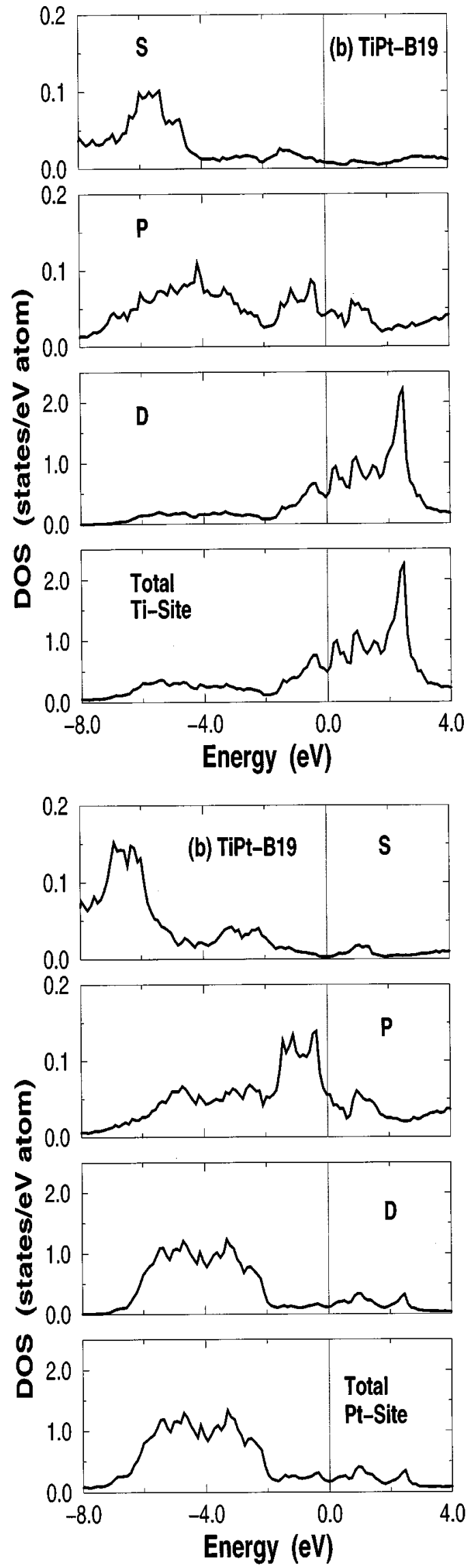

FIG. 12. Site and angular momentum decomposed density of states for TiPt in the $B 19$ structure for (a) Ti site; and (b) Pt site. The scales are not the same in different panels.
TABLE IV. The $B 2$ heat of formation per formula unit $(H(B 2))$, and the differences in heat of formation per formula unit $\Delta H$ between the $B 2$ and the martensitic phase $\left(B 19 / B 19^{\prime}\right)$ are compared with the melting point ( $B 2$ phase) and the $M_{s}$ temperature.

\begin{tabular}{lcccc}
\hline \hline & Melt Temp. $(\mathrm{K})$ & $H(B 2)(\mathrm{eV})$ & $\Delta H(\mathrm{eV})$ & $M_{s}(\mathrm{~K})$ \\
\hline TiNi & 1583 & -0.66 & -0.09 & 333 \\
TiPd & 1673 & -0.92 & -0.19 & 783 \\
TiPt & 1830 & -1.49 & -0.31 & 1343 \\
\hline \hline
\end{tabular}

optimization of energies and agree well with experimental results for TiNi where there has been very detailed experimental measurement. For all three alloys, the relative structural stability of the $B 2$ and $B 19 / B 19^{\prime}$ phase can be correlated with the change of the electronic structure. The electronic properties are rather similar for the three alloys, which is not surprising since $\mathrm{Ni}, \mathrm{Pd}$, and $\mathrm{Pt}$ have the same number of valence electrons. If we look at the fine details, the properties of TiPd and TiPt are closer to each other than to $\mathrm{TiNi}$; this is also not surprising since the $4 d$ metals are usually closer to the $5 d$ metals in behavior than to the $3 d$ metals. In these alloys, the $B 2$ phase density of states has a two peak structure with the lower energy part dominated by the transition metal $d$ states and the higher energy part dominated by the Ti $d$ states, separated by a dip. The DOS at $E_{F}$ is mainly contributed by Ti $d$ states. The $B 19 / B 19^{\prime}$ structure of all three alloys has a lower density of states at $E_{F}$ than the $B 2$ structure. The pseudogap between the transitionmetal $d$ bands and the Ti $d$ bands are diminished and the $d$-band width of both the constituent elements increases, signaling stronger bonding between the two elements in the martensitic phase of these alloys.

Our results also shed some light on the relative stability of the different phases for $\mathrm{Ti}-X$ as we move horizontally across the Periodic Table within the rigid-band picture. Our electronic structure calculations show that if the transition metal $X$ has less electrons per atom than Ni, the DOS at $E_{F}$ should decrease for the $B 2$ phase and the relative stability of the $B 2$ phase should increase. We note that the $B 2$ phase of TiFe is especially stable, it is the only structural phase of equiatomic TiFe that has been documented. ${ }^{2}$ This is consistent with the fact that $\mathrm{Fe}$ has less electrons than $\mathrm{Ni}$, and with an average of six valence electrons per atom, equiatomic TiFe has the same number of valence of atoms as typical bcc metals like $\mathrm{Cr}$, Mo, and $\mathrm{W}$. For the Ti-Ni/Pd/Pt series which we have considered in detail, the average number of electrons per atom is 7 , and we expect a competition between bcc-like $B 2$ structure and the more compact $B 19 / B 19^{\prime}$ structures. For the $\mathrm{Ti}-\mathrm{Cu} / \mathrm{Ag} / \mathrm{Au}$ alloys, with more electrons per atom, the $B 2$ should become even more unstable. It is indeed true that the $B 2$ structure has not been documented for equiatomic Ti alloys of these noble metals. ${ }^{2} \mathrm{The} \mathrm{TiCu}$ and TiAg alloys are found in the $B 19$ structure; and for TiAu, there is a competition between the $B 11$ as the high-temperature phase and the $B 19$ structure. $^{2}$

\section{ACKNOWLEDGMENTS}

We thank Dr. S.A. Shabalovskaya for bringing our attention to this problem and Dr. B.N. Harmon and Dr. Y.G. Ding 
for many stimulating discussions. C.T.C. was supported by RGC Hong Kong through DAG95/96-SC12 from HKUST. Y.Y.Y. is grateful to Ames Lab (USDOE) and HKUST (Physics) for their hospitality, and support by the Chinese National Science Foundation. Ames Laboratory is operated for the U.S. Department of Energy by Iowa State University under Contract No. W-7405-ENG-82. Part of the work has been supported by the Director of Energy Research, Office of Basic Energy Sciences.

\section{APPENDIX}

The elastic constants $c^{\prime}=1 / 2\left(c_{11}-c_{12}\right), c_{11}, c_{12}$, and $c_{44}$ are found by calculating the deformation energy of the NiTi crystal by straining the unit cell. For $c^{\prime}$, the cubic crystal is distorted by the following deformation matrix:

$$
\left(\begin{array}{ccc}
1+\epsilon & 0 & 0 \\
0 & 1+\epsilon & 0 \\
0 & 0 & 1-2 \epsilon
\end{array}\right) .
$$

Now, $\delta E=6 c^{\prime} \epsilon^{2}$. We calculate $\delta E$ for eight pairs of $\pm \epsilon$ and $c^{\prime}$ is found to be $0.12 \times 10^{12} \mathrm{dyn} / \mathrm{cm}^{2}$. Since the bulk modulus $B=\left(c_{11}+2 c_{12}\right) / 3$, knowing $c^{\prime}$ and $B$ also gives $c_{11}$ and $c_{12}$, which are listed in Table I. The value of $c_{44}$ is found by distorting the crystal according to

$$
\left(\begin{array}{lll}
1 & \epsilon & 0 \\
\epsilon & 1 & 0 \\
0 & 0 & 1
\end{array}\right) .
$$

The change of energy is then $\delta E=c_{44} \epsilon^{2} . c_{44}$ is found to be $0.5 \times 10^{12} \mathrm{dyn} / \mathrm{cm}^{2}$. The elastic constant $c^{\prime}$ corresponds to a shear in the [1 $1 \overline{0}]$ direction of the [110] planes. This mode of distortion in the $B 2$ structure is well known to be very soft, as is born out in the calculation, and is frequently associated with the martensitic transformation in this class of alloys. Comparing with experimental results ${ }^{29}$ measured at room temperature, we note that the experimental data, which are temperature dependent, indicate that $c_{44}$ is softer than our calculated result, while $c^{\prime}$ is stiffer than calculated (all our theoretical results correspond to $T=0 \mathrm{~K}$ ). Besides temperature dependent effects, this may also be due to the effects of point defects such as vacancies and antisite defects which can sometimes be present even when the crystal is nominally at stoichiometry. The shear elastic constants $c^{\prime}$ and $c_{44}$ are found to decrease as the temperature is lowered in the temperature range $50-0{ }^{\circ} \mathrm{C}$. Our results indicate that none of the elastic constants, including $c$ ', are "soft" in the $T=0$ limit.
${ }^{1}$ S. Shabalovskaya, A. Narmonev, O. Ivanova, and A. Dementjev, Phys. Rev. B 48, 13296 (1993); S. Shabalovskaya, in ShapeMemory Materials and Phenomena-Fundamental Aspects and Applications, edited by C. T. Liu, H. Kunsmann, K. Otsuka, and M. Wuttig, MRS Symposia Proceedings No. 246 (Materials Research Society, Pittsburgh, 1992), p. 247.

${ }^{2}$ See, e.g., Phase Diagrams of Binary Titanium Alloys, edited by J. L. Murray (ASM International, Metals Park, OH, 1987), and references therein.

${ }^{3}$ See, e.g., P. Hohenberg and W. Kohn, Phys. Rev. 136, B864 (1964); W. Kohn and L.J. Sham, ibid. 140, A1133 (1965); Theory of the Inhomogeneous Electron Gas, edited by N.H. March and S. Lundqvist (Plenum, New York, 1983); R.O. Jones, Rev. Mod. Phys. 61, 689 (1989).

${ }^{4}$ L. Hedin and B.I. Lundqvist, J. Phys. C 4, 2064 (1971).

${ }^{5}$ D.R. Hamann, M. Schlüter, and C. Chiang, Phys. Rev. Lett. 43, 1494 (1979); G.B. Bachelet and M. Schlüter, Phys. Rev. B 25, 2103 (1982).

${ }^{6}$ S.G. Louie, K.M. Ho, and M.L. Cohen, Phys. Rev. B 19, 1774 (1979).

${ }^{7}$ C. Elsasser, N. Takeuchi, K.M. Ho, C.T. Chan, P. Braun, and M. Fahnle, J. Phys. Condens. Matter 2, 4371 (1980).

${ }^{8}$ K.M. Ho, C. Elsässer, C.T. Chan, and M. Fähnle, J. Phys. Condens. Matter 4, 5189 (1992).

${ }^{9}$ C.T. Chan, K.P. Bohnen, and K.M. Ho, Phys. Rev. B 47, 4771 (1993).

${ }^{10}$ K. Otsuka, T. Sawamura, and K. Shimizu, Phys. Status Solidi A 5, 457 (1971). In this article, the coordinates of one of the $\mathrm{Ni}$ atoms are listed as $(1 / 2,2 / 3,1 / 2)$. The number $2 / 3$ should probably be $5 / 6$.

${ }^{11}$ G.M. Michal and R. Sinclar, Acta Crystallogr. Sec. B 37, 1803 (1981)
${ }^{12}$ Y. Kudoh, M. Tokonami, S. Miyazaki, and K. Otsuka, Acta Metall. 33, 2049 (1985).

${ }^{13}$ J.H. Rose, J. Ferrante, and J.R. Smith, Phys. Rev. Lett. 47, 675 (1981).

${ }^{14}$ V.L. Moruzzi, J.F. Janak, and A.R. Williams, Calculated Electronic Properties of Metals (Pergamon, New York, 1978).

${ }^{15} \mathrm{Kudoh}$ et al. remarked that there is a possible misprint in the sign of the Ti coordinates in the article of Michal and Sinclar (MS). The structural parameters quoted under the column "MS" in Table II in this article is taken from Table II of Kudoh et al., which corrects for the possible misprint in sign in the article of MS. We also note that the calculation of Bihlmayer et al. (Ref. 20 ) on the electronic properties of NiTi $B 19^{\prime}$ used the original published $x, y$ coordinates of MS, but $\operatorname{Ti}(z)$ is quoted as 0.75 rather than 0.25 as in the MS paper. This effectively corrects for the misprint in MS original article. However, $\operatorname{Ti}(x)$ is quoted as 0.5247 instead of the 0.5274 in MS paper. The difference is small enough that the results on the electronic structure should not depend upon changes in the third decimal place. The atomic positions given by Kudoh et al. definitely give lower energy than the coordinates of Michal et al. (after correction for the misprint). In addition, the conspicuous dip in the DOS of the $B 19^{\prime}$ structure is obtained with Kudoh's coordinates (which is essentially identical to the theoretical results), but not with coordinates of MS. With the coordinates of MS, there is a shoulder rather than a dip in the Fermi level. The major difference between the coordinates of Kudoh et al. and the coordinates of MS lies in the position of $\operatorname{Ti}(x)$, which is listed as 0.4176 in the paper of Kudoh et al. and quoted as 0.4726 by Kudoh et al. for MS. We note that if there is a misprint in Table I of MS, so that $\operatorname{Ti}(x)$ should be 0.5724 rather than 0.5274 , then the coordinates of MS and Kudoh will match pretty well with each other and 
with the first-principles calculations.

${ }^{16}$ A. Pasturel, C. Colinet, D. Nguyen Manh, A.T. Paxton, and M. van Schilfgaarde, Phys. Rev. B 52, 15176 (1995).

${ }^{17}$ G. Bihlmayer, R. Eibler, and A. Neckel, Phys. Rev. B 50, 13113 (1994)

${ }^{18}$ The curve is calculated with $40 k$ points in the irreducible Brillouin zone. The energy differences between different phases quoted in the text are calculated with four atoms per unit cell (for the best cancellation of errors) and $285 k$ points in the IBZ for $B 19 / B 19^{\prime}, B 2$, and $B 11$, and $216 k$ points for the $L 1_{0}$.

${ }^{19}$ G.L. Zhao and B.N. Harmon, Phys. Rev. B 48, 2031 (1993).

${ }^{20}$ G. Bihlmayer, R. Eibler, and A. Neckel, J. Phys. Condens. Matter 5, 5083 (1993); Ber. Bunsenges. Phys. Chem. 96, 1626 (1992).

${ }^{21}$ S.E. Kul'kova, K.A. Beketov, V.E. Egorushkin, and O.N. Muryzhnikova, J. Phys. (France) IV, Colloq. 5, C8-539 (1995); S.E. Kulkova, V.E. Egorushkin, and V.V. Kalchikhin, Solid State Commun. 77, 667 (1991).

${ }^{22}$ G. Lehmann and M. Taut, Phys. Status Solidi B 54, 469 (1972). The DOS are calculated with $k$ points equivalent to about 20000 $k$ points in the full zone of a $B 2$ structure.

${ }^{23}$ The LDOS are calculated with a LMTO scheme, since the mixedbasis code is currently not equipped with angular decomposition of the DOS. The band structure and the total DOS from the
LMTO code and the mixed-basis code are very similar as long as the same atomic coordinates are used and we expect the same for the angular momentum decomposed LDOS.

${ }^{24}$ T. Saburi, MRS Int'l Mtg. Adv. Mats. 9, 77 (1989).

${ }^{25}$ H.C. Donkersloot and J.H.N. van Vucht, J. Less-Common Met. 20, 83 (1970).

${ }^{26}$ G. Bihlmayer, R. Eibler, and A. Neckel, Philos. Mag. B 73, 511 (1996).

${ }^{27}$ G.W. Fernando, R.E. Watson, and M. Weinert, Phys. Rev. B 45, 8233 (1992).

${ }^{28}$ E. Goo and R. Sinclar, Acta Metall. 33, 1717 (1985); K. Otsvka, T. Sawamura, and K. Shimizu, Phys. Status Solidi A 5, 457 (1971).

${ }^{29}$ O. Mercier, K.N. Melton, G. Gremand, and J.J. Hagi, J. Appl. Phys. 51, 1833 (1980).

${ }^{30} \mathrm{R}$. Hultgren, Selected Values of the Thermodynamic Properties of Binary Alloys (American Society for Metals, Metals Park, OH, 1973); I Barin, D. Knacke, and O. Kubaschewski, Thermochemical Properties of Inorganic Substances (Suppl.) (SpringerVerlag, Berlin, 1977)

${ }^{31}$ A.E. Dwight, R.A. Conner, Jr., and J.W. Downey, Acta Crystallogr. 18, 835 (1965). 